PALLIATIVE CARE

\section{Improved pain management}

The Edinburgh Pain Assessment and management Tool (EPAT) was created for the systematic assessment of cancer-related pain. Now, a study conducted in multiple cancer inpatient units describes how the EPAT could be routinely used to improve the pain-related outcomes of patients with cancer.

The use of the EPAT involves systematic recording of the worst pain (on a $0-10$ scale) that patients have felt since last being assessed. Such measurements are incorporated into an algorithm that facilitates decision-making (including opioid prescription). This tool also involves regular reassessment of pain and opioid-related adverse events.

Of the current 40 cancer inpatient centres in the UK, 19 participated in this study. The primary outcome was change in the percentage of participants with a clinically significant improvement in pain, defined as a $\geq 2$-point reduction in the severity of worst pain reported over the previous $24 \mathrm{~h}$ measured between admission and reassessment (3-5 days after admission). In the first phase of the study, cancer-related pain was managed following clinician's judgement and according to local guidelines (defined as universal care; UC) between assessments. Centres were then randomly assigned to implement the EPAT $(n=10)$, or to keep providing UC $(n=9)$. Data from 50 patients per centre were used for the first phase of the study, and from 1,795 patients in the randomization phase. Patients had one of several cancer types, had a mean age of 60 years, and $49 \%$ were women.

The percentage of participants with a clinically significant improvement in worst pain increased from $47.7 \%$ in the initial phase to $54.1 \%$ after implementing the EPAT, but decreased from an initial $50.6 \%$ to $46.4 \%$ in centres that only provided UC. No difference existed in the percentage of patients who received 'strong' opioids $(80 \%)$, or in the incidence of opioid-related adverse effects. In summary, EPAT is a tool that could be routinely used in to improve the management of pain in patients with cancer.

Diana Romero

ORIGINAL ARTICLE Fallon, M. et al. Pain management in cancer center inpatients: a cluster randomized trial to evaluate a systematic integrated approach — the Edinburgh Pain Assessment and management Tool. J. Clin. Oncol. https:// doi.org/10.1200/JCO.2017.76.1825 (2018)

\title{
PET imaging reveals EGFR mutation status
}

Targeted therapies have transformed the outcomes of patients with non-small-cell lung cancer (NSCLC), although only a minor subset of tumours harbours targetable alterations. Now, researchers have developed a PET tracer with a high level of specificity for activating EGFR mutations.

Researchers synthesized a PEGylated anilinoquinazoline derivative (MPG), which was then radiolabelled to enable detection using PET imaging $\left({ }^{18} \mathrm{~F}-\mathrm{MPG}\right)$. Preliminary experiments involving NSCLC-derived cell lines confirmed the in vitro selectivity of this imaging probe for cells harbouring activating EGFR mutations, including a $90 \%$ decrease in signal in an EGFR-mutation-positive cell line pretreated with the EGFR inhibitor gefitinib. In vivo studies in mouse models confirmed the safety and metabolic stability of ${ }^{18} \mathrm{~F}-\mathrm{MPG}$.

In patients with NSCLC, ${ }^{18} \mathrm{~F}-\mathrm{MPG}$ was found to selectively accumulate in tumours harbouring EGFR-activating mutations, relative to $E G F R$-wild-type tumours $\left(F_{2,67}=16.30 ; P<0.0001\right)$. Applying a standard uptake value ( $\left.\mathrm{SUV}_{\max }\right)$ cut-off of 2.23 enabled the discrimination of EGFR-mutant-positive

\section{tumours from their EGFR-negative} counterparts with a sensitivity of $86.5 \%$ and a specificity of $81.8 \%$. A concordance of $84.3 \%$ was observed between ${ }^{18} \mathrm{~F}-\mathrm{MPG}$ using this threshold and amplification refractory mutation system (ARMS)-PCR of tumour biopsy samples for determination of EGFR mutation status.

Among 38 patients who received EGFR-tyrosine-kinase inhibitors on the basis of a tumour $\mathrm{SUV}_{\max }>2.23$ on ${ }^{18} \mathrm{~F}-\mathrm{MPG}$ imaging, the objective response rate was $81.6 \%$, versus $46.5 \%$ in unselected patients. In one patient, who had stable disease on gefitinib for 20 months, $\mathrm{SUV}_{\max }$ was 2.31 after 6 months of treatment. Following disease regression, owing to biopsy-confirmed conversion to an EGFR-wild-type phenotype, $\mathrm{SUV}_{\text {max }}$ decreased to 2.01, suggesting sensitivity for acquired resistance. These promising findings warrant prospective validation in patients with NSCLC.

Peter Sidaway

ORIGINAL ARTICLE Sun, X. et al. A PET imaging approach for determining EGFR mutation status for improved lung cancer patient management. Sci. Transl Med. 10, eaan8840 (2018)

\section{UROLOGICAL CANCER}

\section{DDR signature to predict response to $\mathrm{ICl}$}

A subset of urothelial carcinomas harbour alterations in genes involved in DNA-damage response and repair (DDR). In addition to an increased mutational burden, these tumours are characterized by higher objective response rates (ORR) to platinum-based therapies. Jonathan Rosenberg and colleagues have examined the response of patients with this tumour subset to immune-checkpoint inhibitors (ICls), with results now published.

This retrospective study included tumour samples obtained from 60 patients with metastatic urothelial carcinoma before receipt of $\mathrm{ICl}$ in three different clinical trials. Tumour analysis was performed using MSK-IMPACT, a next-generation sequencing platform consisting of $>341$ genes, including 34 DDR-related genes. Alterations in DDR-related genes were detected in 28 patients (median 1 alteration per patient; range, 1-34 alterations per patient). These alterations were either known or likely to be deleterious in 15 patients ( $25 \%$ ), and of unknown significance in 13 patients (22\%).

"We observed that alterations in DDR genes are significantly associated with clinical benefit in patients receiving single-agent anti-PD1/PDL1 therapy," explains Rosenberg. The presence of a deleterious DDR alteration was associated with an ORR of $80 \%$, and the median PFS duration was not reached, compared with $53.9 \%$ and 15.7 months, respectively, for patients with alterations of unknown significance, and $18.8 \%$ and 2.9 months, respectively, for those without DDR alterations. The lack of predictive biomarkers of response to anti-PD1/PDL1 therapy is an unmet need in the management of patients with metastatic urothelial carcinoma and other cancers. "In our data set, the presence of DDR alterations was better at predicting clinical responses than mutation burden, suggesting that additional mechanisms might be at play. In addition, mutation burden is a continuous variable, whereas DDR mutations are a categorical variable, and might be more easily applied to each individual patient," concludes Rosenberg, who is planning to validate the DDR signature as a biomarker in prospective studies.

Diana Romero

ORIGINAL ARTICLE Teo, M. Y. et al. Alterations in DNA damage response and repair genes as potential marker of clinical benefit from PD-1/PD-L1 blockade in advanced urothelial cancers.J. Clin. Oncol. https://doi.org/10.1200/ JCO.2017.75.7740 (2018) 